\title{
Adenosine monophosphate-activated protein kinase activation mediates the leptin-induced attenuation of cognitive impairment in a streptozotocin-induced rat model
}

\author{
BIN ZHU ${ }^{1}$, RI-YUE JIANG ${ }^{2}$, CHUN YANG $^{3}$ and NING LIU ${ }^{1}$ \\ Departments of ${ }^{1}$ Critical Care Medicine, ${ }^{2}$ Radiotherapy and ${ }^{3}$ Anesthesiology, \\ The Third Affiliated Hospital of Soochow University, Changzhou, Jiangsu 213003, P.R. China
}

Received June 25, 2014; Accepted February 16, 2015

DOI: $10.3892 /$ etm.2015.2317

\begin{abstract}
Several lines of evidence have shown that the incidence of cognitive impairment in diabetic patients is significantly higher than that in healthy individuals, but the exact pathogenesis has not been fully elucidated. Furthermore, it has been suggested that leptin may have a therapeutic effect in cognitive dysfunction. The aim of the present study was to observe the effect of leptin on cognitive dysfunction in streptozotocin (STZ)-induced diabetic rats, and to explore whether adenosine monophosphate-activated protein kinase (AMPK) activation was involved in any potential therapeutic effect of leptin. Compared with control rats, STZ rats exhibited decreased levels of AMPK and a poor performance in the Morris water maze, while these changes were reversed by leptin. Furthermore, Compound C, an AMPK antagonist, significantly attenuated the leptin-induced cognitive function improvement in the STZ rats. In conclusion, these results suggest that AMPK activation may play a critical role in the leptin-induced attenuation of STZ-induced cognitive impairment.
\end{abstract}

\section{Introduction}

Diabetes mellitus is a common disease in older individuals, affecting $\sim 20 \%$ of the population aged $>65$ years $(1,2)$. It has been reported that diabetes has a close association with the reduced performance in numerous domains of cognitive function (1,3-5). A clinical study by Arvanitakis et al (1) has indicated that diabetes may be associated with an increased risk of Alzheimer's disease development and may eventually affect cognitive function; however, the underlying mechanisms

Correspondence to: Dr Chun Yang, Department of Anesthesiology, The Third Affiliated Hospital of Soochow University, 185 Juqian Street, Changzhou, Jiangsu 213003, P.R. China

E-mail: yangchuntz@sina.com

Key words: diabetes, leptin, cognitive impairment, adenosine monophosphate-activated protein kinase, streptozotocin of diabetes-induced cognitive dysfunction have not been fully elucidated.

Numerous studies have focused on diabetes-induced cognitive impairment, while the majority have focused on fat metabolism and vascular dementia (6-8). Jafari Anarkooli et al (9) demonstrated that Bcl-2 family gene expression and caspase-3 activity were altered in the streptozotocin (STZ)-induced diabetic rat hippocampus. Furthermore, Revsin et al (10) found that a glucocorticoid receptor antagonist normalized hippocampal alterations and cognitive impairment in STZ-induced diabetic mice. Our previous study (11), however, found that intraperitoneal injection of a single dose of $60 \mathrm{mg} / \mathrm{kg}$ STZ could cause cognitive dysfunction and increase inflammatory cytokine levels and oxidative activity in the rat hippocampus. Notably, leptin levels in the rat hippocampus significantly decreased.

Leptin, which is a hormone-like protein secreted from fat cells, plays a critical role in regulating food intake and energy metabolism $(12,13)$. It has been reported that leptin is a potential cognitive enhancer (14). Several studies have shown that leptin is the upstream activator of the adenosine monophosphate-activated protein kinase (AMPK) pathway $(15,16)$. Yi et al (17) observed that acute and chronic exercise could indirectly activate the leptin-AMPK signaling pathway, while a study by Namkoong et al (18) showed that leptin and insulin deficiencies in diabetes led to increased hypothalamic AMPK activity.

The aim of the present study was to investigate the effect of leptin on cognitive dysfunction in STZ-induced diabetic rats and to explore whether AMPK activation was involved in any potential therapeutic effects of leptin. An AMPK antagonist, Compound $\mathrm{C}$, was selected to investigate the potential involvement of AMPK in the leptin-induced therapeutic effect in cognitive impairment.

\section{Materials and methods}

Animals. Male Wistar rats weighing 220-300 g were purchased from the Shanghai Animal Center (Shanghai, China). Six rats were housed per cage with food and water available ad libitum and maintained under a 12-h light/dark cycle (lights on at 7:00 a.m.). Animal care was approved by the Animal Use and 
Protection Committee of Soochow University (Changzhou, China). Thirty-six rats were randomly divided into three groups $(\mathrm{n}=12$ each). The rats were intraperitoneally pretreated with either a single injection of saline or STZ (Sigma-Aldrich, St. Louis, MO, USA) at a dose of $60 \mathrm{mg} / \mathrm{kg}$. One month later, the rats were either intracerebroventricularly injected with saline or $10 \mu \mathrm{g}$ leptin (Sigma-Aldrich) dissolved in $5 \mu \mathrm{l}$ Tris-HCl. For the second experiment, a further 36 rats were randomly divided into three groups ( $n=12$ each). The rats were intraperitoneally pretreated with either a single injection of saline or STZ at a dose of $60 \mathrm{mg} / \mathrm{kg}$ according to the first protocol. One month later, the rats were either intracerebroventricularly injected with saline, $10 \mu \mathrm{g}$ leptin or $10 \mu \mathrm{g}$ leptin plus intraperitoneal injection of $1 \mathrm{mg} / \mathrm{kg}$ Compound C (Tocris Bioscience, Bristol, UK).

Morris water maze test. According to our previous study (11), cognitive function was assessed using the Morris water maze test system between 9:00 a.m. and 3:00 p.m. The maze was $80 \mathrm{~cm}$ deep and $100 \mathrm{~cm}$ in diameter and was filled with water to a depth of $30 \mathrm{~cm}$. The maze was divided into four quadrants of equal size on the monitor screen of a computer, and the water temperature was maintained at $23-24^{\circ} \mathrm{C}$. The swimming paths of the rats were recorded by a video camera and analyzed by VideoMot software (Huaibei Zhenghua Biologic Apparatus Facilities Co., Ltd., Huaibei, China). The trials were conducted for four consecutive days in order to observe the escape latency of the rats and the time spent in each quadrant. The escape latency and the proportion of time spent in the target quadrant were recorded.

Leptin and tumor necrosis factor- $\alpha(T N F-\alpha)$ measurement. Leptin and TNF- $\alpha$ levels were determined using ELISA kits. According to the manufacturer's instructions (Wuhan Huamei Bioengineering Co., Ltd., Wuhan, China), microtiter plates were coated for overnight incubation with the samples diluted 1:2 in sample diluent. The plates were then washed three times with sample diluent, and the primary antibody (monoclonal anti-leptin or -TNF- $\alpha$ ), diluted 1:1,000 in sample diluent, was added to each well and incubated for $3 \mathrm{~h}$ at room temperature. Subsequent to washing, a peroxidase-conjugated anti-rabbit antibody (diluted 1:10,000; Nanjing Sunshine Biotechnology Co., Ltd.) was added to each well and incubated at room temperature for $1 \mathrm{~h}$. Streptavidin enzyme, substrate and stop solution were then added, and the leptin and TNF- $\alpha$ levels were determined by measuring the absorbance at $450 \mathrm{~nm}$. In addition, total protein was measured using the Lowry method with bovine serum albumin as a standard.

Amyloid- $\beta(A \beta)$ measurement. The animals were sacrificed immediately by decapitation and the protein concentrations were determined using a bicinchoninic acid assay kit (cat. no. P0012S; Beyotime Institute of Biotechnology, Haimen, China). The samples were then centrifuged at 3,000 x g at $4^{\circ} \mathrm{C}$ for $30 \mathrm{~min}$ to obtain the supernatants. The proteins were separated using SDS-PAGE and transferred onto polyvinylidene difluoride membranes. Subsequent to blocking with 5\% non-fat milk, the membranes were incubated with rabbit anti-A $\beta$ primary antibody (1:200; Sigma-Aldrich). The membranes were then incubated for $1 \mathrm{~h}$ at room temperature with anti-rabbit horseradish peroxidase-conjugated immunoglobulin G
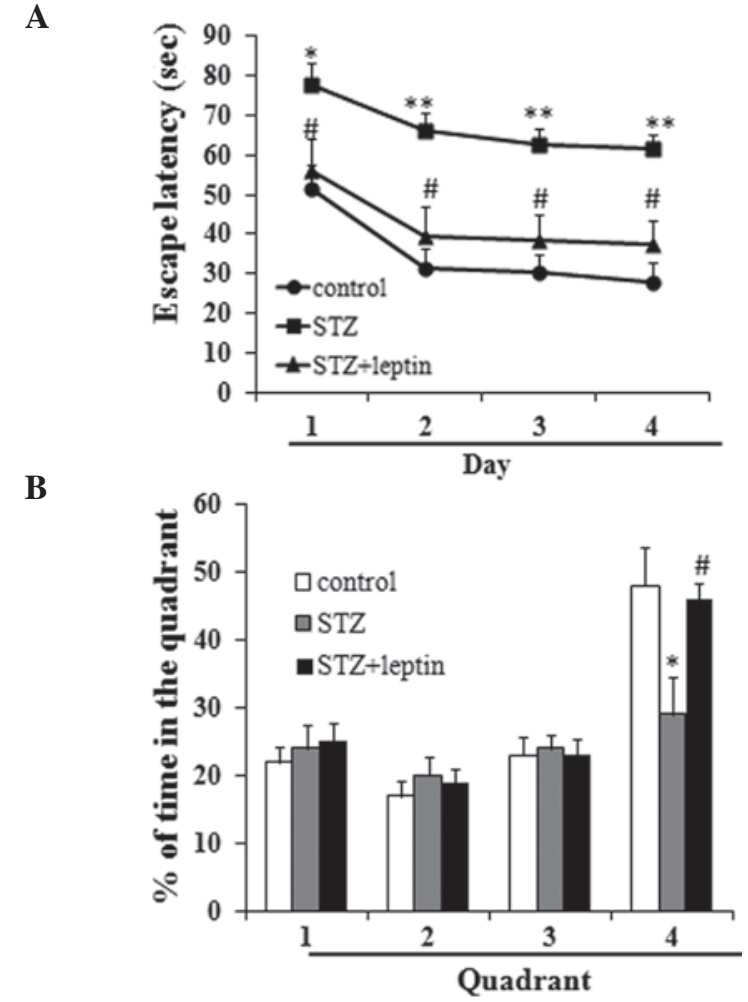

Figure 1. Effect of STZ and/or leptin on (A) the escape latency of the rats and $(\mathrm{B})$ the proportion of time spent in the target quadrant in the Morris water maze test. ${ }^{*} \mathrm{P}<0.05$ and ${ }^{* *} \mathrm{P}<0.01$ vs. control; ${ }^{\text {}} \mathrm{P}<0.05$ vs. STZ. STZ, streptozotocin.

secondary antibody (1:20,000; CWBIO, Beijing, China). The labeled protein was detected using enhanced chemiluminescence reagents (GE Healthcare Life Sciences, Little Chalfont, UK) and the band intensity was analyzed using ImageJ software (National Institutes of Health, Bethesda, MD, USA).

Malondialdehyde (MDA) measurement. According to our previous study (11), the samples were mixed with $1 \mathrm{ml} \mathrm{10 \%}$ trichloroacetic acid and $1 \mathrm{ml} 0.67 \%$ thiobarbituric acid, and were then heated in a boiling water bath for $30 \mathrm{~min}$. MDA equivalents were determined in the tissue and submitochondrial particles of the rat brain using a spectrophotometer at an absorbance of $532 \mathrm{~nm}$.

Blood glucose measurement. The rats were anesthetized with $10 \%$ chloral hydrate $(0.4 \mathrm{ml} / 100 \mathrm{~g}$, intraperitoneal $)$ and blood samples were then collected from the carotid artery. Blood glucose levels were determined with a blood glucometer (OneTouch ${ }^{\circledR}$ UltraEasy $^{\circledR}$; Johnson \& Johnson, Inc., New Brunswick, NJ, USA).

Statistical analysis. Data are expressed as the mean \pm standard deviation. Statistical analyses were performed by one-way analysis of variance, and post hoc analyses were conducted using Fisher's least significant difference tests. All statistical analyses were carried out using SPSS version 17.0 software (SPSS, Inc., Chicago, IL, USA). In the Morris water maze test, the percentage of time spent in the target quadrant was evaluated using $\chi^{2}$ tests. $\mathrm{P}<0.05$ was considered to indicate a statistically significant difference. 


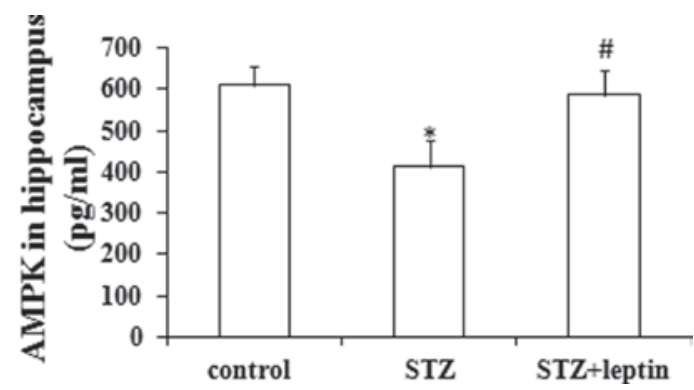

Figure 2. Effect of STZ and/or leptin on the expression of AMPK in the rat hippocampus. ${ }^{*} \mathrm{P}<0.05$ vs. control; ${ }^{*} \mathrm{P}<0.05$ vs. STZ. STZ, streptozotocin; AMPK, adenosine monophosphate-activated protein kinase.

A

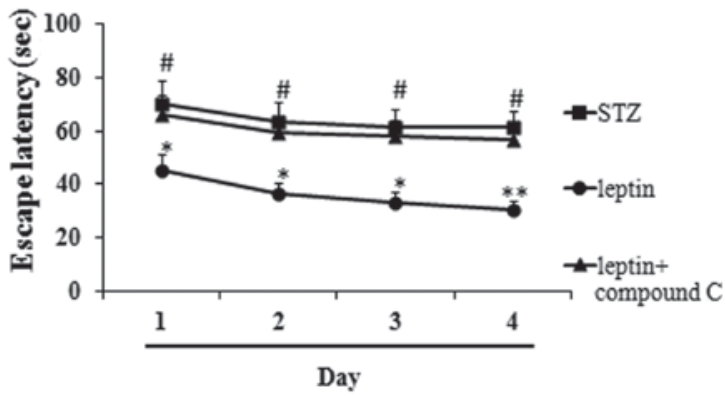

B

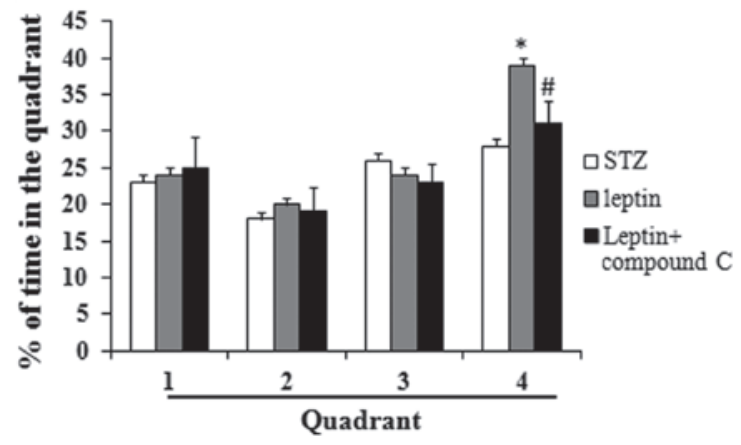

Figure 3. Effect of Compound C on (A) the escape latency of the STZ-induced diabetic rats and (B) the proportion of time spent in the target quadrant in the Morris water maze test. ${ }^{*} \mathrm{P}<0.05$ and ${ }^{* *} \mathrm{P}<0.01$ vs. $\mathrm{STZ}$; ${ }^{~} \mathrm{P}<0.05$ vs. leptin. STZ, streptozotocin.

\section{Results}

Effect of STZ and/or leptin on the escape latency of the rats and the proportion of time spent in the target quadrant in the Morris water maze test. The results of the Morris water maze test demonstrated that STZ administration significantly increased the escape latency as compared with the control group, and decreased the percentage of time spent in the fourth quadrant $(\mathrm{P}<0.05$ or 0.01$)$. Compared with the STZ group, however, rats in the STZ plus leptin group exhibited significantly decreased escape latencies, and an increased percentage of time spent in the fourth quadrant $(\mathrm{P}<0.05)$ (Fig. 1).

Effect of STZ and/or leptin on the expression of AMPK in the rat hippocampus. A single injection of STZ significantly decreased the protein expression of AMPK in the rat hippocampus $(\mathrm{P}<0.05)$. By contrast, leptin administration could abrogate the STZ-induced decrease in AMPK expression in the hippocampus $(\mathrm{P}<0.05)$ (Fig. 2).
A

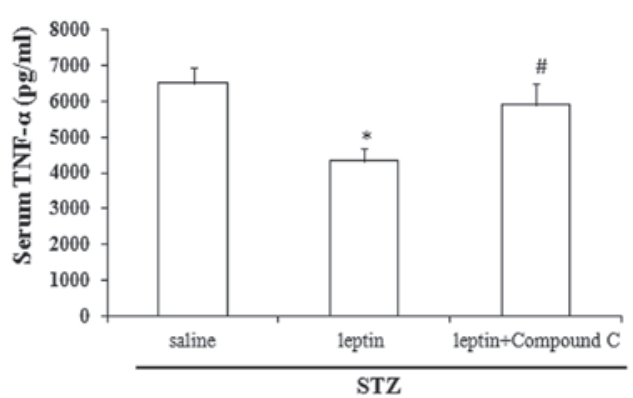

B

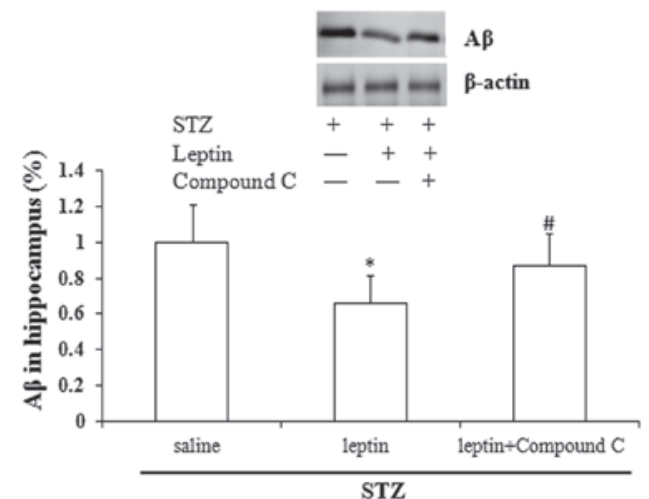

C

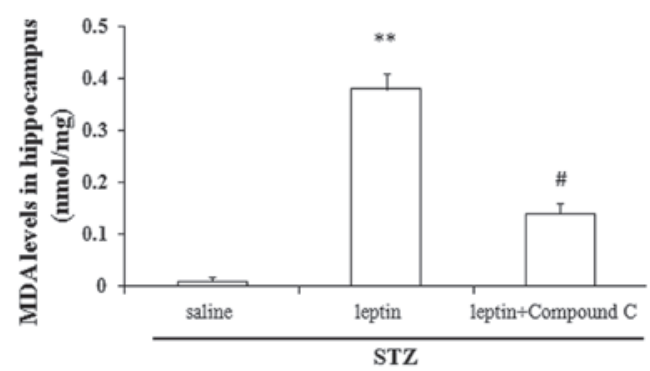

D

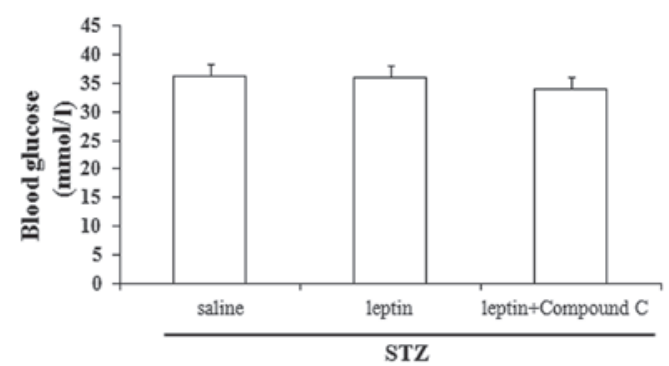

Figure 4. Effect of Compound $\mathrm{C}$ on the leptin-induced changes in the expression of (A) TNF- $\alpha$, (B) A $\beta$, (C) MDA and (D) blood glucose. ${ }^{*} \mathrm{P}<0.05$ and ${ }^{* * *} \mathrm{P}<0.01$ vs. saline; ${ }^{\#} \mathrm{P}<0.05$ vs. leptin. STZ, streptozotocin; TNF- $\alpha$, tumor necrosis factor- $\alpha$; $\beta \beta$, amyloid- $\beta$; MDA, malondialdehyde.

Effect of Compound $C$, an AMPK inhibitor, on the escape latency of STZ-induced diabetic rats and the percentage of time spent in the target quadrant in the Morris water maze test. Compound $\mathrm{C}$, which acts as an AMPK inhibitor, significantly increased the escape latency as compared with the leptin group, and decreased the percentage of time spent in the fourth quadrant $(\mathrm{P}<0.05$ or 0.01$)$ (Fig. $3 \mathrm{~A}$ and $\mathrm{B})$.

Effect of Compound $C$ on the leptin-induced changes in the expression of TNF- $\alpha, A \beta$ and MDA and the blood glucose levels. Compared with the saline group, leptin administration caused a significant decrease in the serum TNF- $\alpha$ and 
$\mathrm{A} \beta$ levels in the rat hippocampus $(\mathrm{P}<0.05)$, and a significant increase in the MDA levels $(\mathrm{P}<0.01)$. Compared with the leptin group, Compound $\mathrm{C}$ abrogated the leptin-induced changes in the expression of TNF- $\alpha, A \beta$ and MDA $(\mathrm{P}<0.05)$, but did not affect the blood glucose levels ( $\mathrm{P}>0.05)$ (Fig. 4A-D).

\section{Discussion}

In the present study, the results demonstrated that leptin could attenuate STZ-induced cognitive impairment, which was characterized by the poor performance of the rats in the Morris water maze test. Compound C, an AMPK inhibitor, significantly abrogated the protective effects of leptin against the STZ-induced rat cognitive impairment. The findings of these results suggested, therefore, that AMPK activation contributed to the underlying mechanism of the therapeutic effect of leptin in STZ-induced cognitive impairment.

Numerous studies have shown that the mechanism of cognitive dysfunction in STZ-induced diabetic rats is associated with the vascular lesions caused by diabetes $(8,19)$. Denver et al (12), however, proposed that diabetes is simply a trigger in the pathogenesis of diabetes-induced cognitive dysfunction, rather than the main mechanism. Zhu et al (20) suggested that oxidative stress, the inflammatory response and $\mathrm{A} \beta$ formation comprised the primary mechanism in diabetes-induced cognitive dysfunction, which was also consistent with the results of our previous study (11). In the present study, the results supported the above-mentioned hypothesis that diabetes-induced cognitive dysfunction manifested as a result of the abnormal expression of TNF- $\alpha, A \beta$ and MDA. Notably, the blood glucose levels did not show any significant change following leptin treatment. Although it has previously been demonstrated that leptin acts to reduce blood glucose levels (21), the present study utilized an injection of exogenous leptin, not endogenous; therefore, the leptin was not able to rapidly activate the leptin receptors, which prevented it from exerting its biological effects.

AMPK is an enzyme that plays a role in cellular energy homeostasis. Numerous studies have highlighted the important role of AMPK in the pathogenesis of Alzheimer's disease, which is characterized by cognitive impairment $(22,23)$. An in vitro study by Greco et al (24) showed that leptin increased cellular metabolism by activating AMPK to reduce $A \beta$ expression in neurons. A different study also performed by this group (25) demonstrated that leptin directly regulated $A \beta$ through the AMPK pathway. The results of the present study showed that STZ administration significantly decreased the AMPK levels in the rat hippocampus, while leptin abrogated this effect. Notably, it was observed that Compound C, an AMPK inhibitor, significantly reversed the effects of leptin, suggesting that AMPK activation is likely to be involved in the mechanism underlying the protective effect of leptin against cognitive impairment.

The activation of oxidative stress has been considered to be an important inducing factor for the incidence of cognitive impairment. Praticò et al (26) demonstrated that increased oxidative stress could be used as a predictor for the onset of cognitive impairment. A different study suggested that oxidative activation has harmful effects on the rat synapses in the cerebral cortex and hippocampus, which may result in cognitive impairment (27). Notably, a clinical study found that the successful treatment of patients with cognitive impairment could restore the oxidative cytokine levels in the peripheral blood of the patients to a normal levels (28). In the present study, it was observed that an AMPK inhibitor could abrogate the effect of leptin on cognitive impairment and change hippocampal MDA levels. This result indirectly showed that the control of cognitive function by AMPK signaling potentially occurs through the regulation of oxidative stress. Similarly, the inflammatory response plays a critical role in the incidence of diabetes-related cognitive impairment. The present results showed that TNF- $\alpha$ levels decreased following leptin treatment, while the AMPK inhibitor reversed this change. This result was consistent with our expected hypothesis.

In conclusion, AMPK activation may contribute to the therapeutic effect of leptin in cognitive impairment in an STZ-induced rat model. It is likely that oxidative stress and the inflammatory response are also involved.

\section{References}

1. Arvanitakis Z, Wilson RS, Bienias JL, Evans DA and Bennett DA: Diabetes mellitus and risk of Alzheimer disease and decline in cognitive function. Arch Neurol 61: 661-666, 2004.

2. Engelgau MM, Geiss LS, Saaddine JB, et al: The evolving diabetes burden in the United States. Ann Intern Med 140: 945-950, 2004.

3. van den Berg E, Reijmer YD, de Bresser J, et al; Utrecht Diabetic Encephalopathy Study Group: A 4 year follow-up study of cognitive functioning in patients with type 2 diabetes mellitus. Diabetologia 53: 58-65, 2010.

4. Huber JD: Diabetes, cognitive function, and the blood-brain barrier. Curr Pharm Des 14: 1594-1600, 2008.

5. Strachan MW, Price JF and Frier BM: Diabetes, cognitive impairment, and dementia. BMJ 336: 6, 2008.

6. Peila R, Rodriguez BL and Launer LJ; Honolulu-Asia Aging Study: Type 2 diabetes, APOE gene and the risk for dementia and related pathologies: The Honolulu-Asia Aging Study. Diabetes 51: 1256-1262, 2002.

7. Breteler MM: Vascular risk factors for Alzheimer's disease: An epidemiologic perspective. Neurobiol Aging 21: 153-160, 2000.

8. Gispen WH and Biessels GJ: Cognition and synaptic plasticity in diabetes mellitus. Trends Neurosci 23: 542-549, 2000.

9. Jafari Anarkooli I, Sankian M, Ahmadpour S, Varasteh AR and Haghir H: Evaluation of Bcl-2 family gene expression and Caspase-3 activity in hippocampus STZ-induced diabetic rats. Exp Diabetes Res 2008: 638467, 2008.

10. Revsin Y, Rekers NV, Louwe MC, et al: Glucocorticoid receptor blockade normalizes hippocampal alterations and cognitive impairment in streptozotocin-induced type 1 diabetes mice. Neuropsychopharmacology 34: 747-758, 2009.

11. Yang C, Zhu B, Ding J and Wang ZG: Isoflurane anesthesia aggravates cognitive impairment in streptozotocin-induced diabetic rats. Int J Clin Exp Med 7: 903-910, 2014.

12. Denver RJ, Bonett RM and Boorse GC: Evolution of leptin structure and function. Neuroendocrinology 94: 21-38, 2011.

13. Singh A, Wirtz M, Parker N, et al: Leptin-mediated changes in hepatic mitochondrial metabolism, structure and protein levels. Proc Natl Acad Sci USA 106: 13100-13105, 2009.

14. Harvey J, Shanley LJ, O'Malley D and Irving AJ: Leptin: A potential cognitive enhancer? Biochem Soc Trans 33: 1029-1032, 2005.

15. Minokoshi Y, Kim YB, Peroni OD, et al: Leptin stimulates fatty-acid oxidation by activating AMP-activated protein kinase. Nature 415: 339-343, 2002.

16. Minokoshi Y and Kahn BB: Role of AMP-activated protein kinase in leptin-induced fatty acid oxidation in muscle. Biochem Soc Trans 31: 196-201, 2003.

17. Yi X, Cao S, Chang B, et al: Effects of acute exercise and chronic exercise on the liver leptin-AMPK-ACC signaling pathway in rats with type 2 diabetes. J Diabetes Res 2013: 946432, 2013. 
18. Namkoong C, Kim MS, Jang PG, et al: Enhanced hypothalamic AMP-activated protein kinase activity contributes to hyperphagia in diabetic rats. Diabetes 54: 63-68, 2005.

19. Sharma B and Singh N: Attenuation of vascular dementia by sodium butyrate in streptozotocin diabetic rats. Psychopharmacology (Berl) 215: 677-687, 2011.

20. Zhu X, Raina AK, Lee HG, Casadesus G, Smith MA and Perry G: Oxidative stress signalling in Alzheimer's disease. Brain Res 1000: 32-39, 2004.

21. Davis C, Mudd J and Hawkins M: Neuroprotective effects of leptin in the context of obesity and metabolic disorders Neurobiol Dis 72: 61-71, 2014.

22. Salminen A, Kaarniranta K, Haapasalo A, Soininen H and Hiltunen M: AMP-activated protein kinase: A potential player in Alzheimer's disease. J Neurochem 118: 460-474, 2011.

23. Cai Z, Yan LJ,Li K, Quazi SH and Zhao B: Roles of AMP-activated protein kinase in Alzheimer's disease. Neuromolecular Med 14: $1-14,2012$.
24. Greco SJ, Sarkar S, Johnston JM and Tezapsidis N: Leptin regulates tau phosphorylation and amyloid through AMPK in neuronal cells. Biochem Biophys Res Commun 380: 98-104, 2009.

25. Greco SJ, Hamzelou A, Johnston JM, Smith MA, Ashford JW and Tezapsidis N: Leptin boosts cellular metabolism by activating AMPK and the sirtuins to reduce tau phosphorylation and $\beta$-amyloid in neurons. Biochem Biophys Res Commun 414: 170-174, 2011.

26. Praticò D, Clark CM, Liun F, Rokach J, Lee VY and Trojanowski JQ: Increase of brain oxidative stress in mild cognitive impairment: a possible predictor of Alzheimer disease. Arch Neurol 59: 972-976, 2002.

27. Fukui K, Omoi NO, Hayasaka T, et al: Cognitive impairment of rats caused by oxidative stress and aging and its prevention by vitamin E. Ann NY Acad Sci 959: 275-284, 2002.

28. Padurariu M, Ciobica A, Hritcu L, Stoica B, Bild W and Stefanescu C: Changes of some oxidative stress markers in the serum of patients with mild cognitive impairment and Alzheimer's disease. Neurosci Lett 469: 6-10, 2010. 